\title{
Application of a disk specimen loaded according to the "Brazilian test" for evaluating the brittle strength of materials of non-geological origin
}

\author{
V.Yu. Goltsev, A.V. Osintsev ${ }^{\dagger}$, A.S. Plotnikov \\ †AVOsintsev@yandex.ru \\ National research nuclear university “MEPhI”, Kashirskoe shosse 31, Moscow, Russia, 115409
}

\begin{abstract}
Basing on finite elements method simulations by means of ANSYS software of version 14.0 and experiments using the method of digital image correlation the stress and strain distributions in surface points of a disk specimen loaded according to the "Brazilian test" (compression of the disk in the diametrical plane) have been studied. On the general background of compressive stresses, the presence of tensile stresses in the axial plane of the disk has been detected. These stresses arise in the zone of contact between the sample and loading surfaces and displaces then to the disk center with an increase of external load. The dependences of the maximum tensile stress, tensile stress in the center of the disk and in the center of its surface on the stress parameter determined by the formula recommended by ASTM are obtained. Acceptable ratios between the specimen diameter and thickness for a correct determination of ultimate tensile stress are determined. Results of the numerical analysis are compared with test results carried out on cast iron and graphite specimens. Specimens failed in the plane of tensile stresses action. Difference in the character of fracture of cast iron and graphite specimens is identified, which consists in the fact that fracture of cast iron specimens is ductile while it is brittle for graphite. Good agreement between numerical and experimental results when using the formula provided by ASTM standard to determine the ultimate tensile stress of rock materials was obtained. Possibility of application of the method of testing small samples under the "Brazilian test" for indirect estimation of tensile strength of brittle materials of a non-geological origin is confirmed.
\end{abstract}

Kewords: disk specimen, Brazilian test, strength of the material in tension, small specimens.

\section{Применение дискового образца, нагружаемого по схеме «бразильского теста», для оценки хрупкой прочности материалов негеологического происхождения}

\author{
Гольцев В.Ю., Осинцев А.В. ${ }^{\dagger}$, Плотников А.С. \\ Национальный исследовательский ядерный университет «МИФИ», Каширское шоссе 31, Москва, Россия, 115409
}

\begin{abstract}
Проведены расчетное, с помощью метода конечных элементов, реализованного в программном комплексе ANSYS версии 14.0, и экспериментальное, с применением метода цифровой корреляции изображений, исследования распределения напряжений и деформаций в поверхностных точках дискового образца, нагружаемого по схеме «бразильского теста» (сжатия диска в диаметральной плоскости). На общем фоне сжимающих напряжений отмечено наличие растягивающих напряжений в осевой плоскости диска, возникающих сначала в зоне контакта образца с нагружающими поверхностями и смещающихся от контактной зоны к центру диска при возрастании нагрузки. Получены зависимости максимальных растягивающих напряжений и растягивающих напряжений в середине диска и на его поверхности в центре от параметра напряжения, определяемого по рекомендуемой ASTM формуле. Выявлены допустимые соотношения диаметра и толщины образца для определения достоверных значений прочности материала при испытании. Результаты расчетного анализа сопоставлены с результатами экспериментального исследования, проведенного на образцах из серого чугуна и графита. Разрушение испытанных образцов происходило в плоскости действия растягивающих напряжений. Выявлено различие в характере разрушения чугунных образцов и образцов из графита - пластичное разрушение чугунных образцов и хрупкое разрушение графитовых. Показано хорошее соответствие результатов расчета и эксперимента при использовании расчетной формулы, рекомендованной Стандартом ASTM, для определения хрупкой прочности материалов горных пород. Подтверждена возможность применения метода испытания малоразмерных образцов по схеме «бразильского теста» для косвенной оценки прочности на растяжение хрупких материалов негеологического происхождения.
\end{abstract}

Ключевые слова: дисковый образец, бразильский тест, прочность материала при растяжении, малоразмерные образцы. 


\section{1. Введение}

Отработка технологии производства современных материалов, режимов сварки и спекания $[1,2]$ осуществляется, как правило, на малых образцах, размер которых часто не превышает 10-15 мм в диаметре, а толщина может варьироваться от 1 до 10 мм. Стандартные методы механических испытаний к таким образцам часто не применимы. Для оценки механических свойств материала приходится прибегать к альтернативным методам испытания. Примером таких методов могут быть изгиб тонких дисков на кольцевой опоре $[3,4]$ и сжатие диска в диаметральной плоскости по схеме «бразильского теста» $[5,6]$. Эти методы используются для испытания хрупких материалов. В частности, испытание по схеме «бразильского теста» применяется для определения прочности на растяжение горных пород. Целью настоящей работы является исследование возможности применения испытания малоразмерных образцов по схеме «бразильского теста» для оценки прочности на растяжение хрупких материалов негеологического происхождения.

Согласно ASTM D3967-95a, предел прочности на растяжение хрупких материалов горных пород может быть установлен при испытаниях на диаметральное сжатие образца в виде диска диаметром не менее 50 мм с отношением толщины к диаметру в диапазонах $0,2 \div 0,75$. Для исследования возможности применения такого метода испытания к определению предела прочности на растяжение хрупких материалов негеологического происхождения (графит, керамики, сплавы металлов) было проведено численное, методом конечных элементов (МКЭ), моделирование процесса сжатия диска и выполнен с помощью метода цифровой корреляции изображений (ЦКИ) экспериментальный анализ результатов испытания образцов из чугуна и графита.

\section{2. Параметры расчётной модели}

Моделирование процесса нагружения дискового образца производилось в верифицированном $[7,8]$ расчётном комплексе ANSYS Mechanical версии 16.2. Расчётная модель представляет собой основной элемент симметрии сжимаемого диска - 1/8 часть. Толщина диска варьировалась от 1 до 10 мм при неизменном диаметре 10 мм. Расчётная модель содержала приблизительно 332 тыс. узлов в 80 тыс. гексаэдрических элементах второго по- рядка (с квадратичными функциями формы) для диска размера $10 \times 5$ мм. Материал модели предполагается идеально-упругим. Модуль упругости материала принят равным 100 ГПа. На модель наложены граничные условия симметрии на плоскостях отсечения расчётной области от полной модели. Нагружение модели производилось сдавливанием абсолютно жёсткой плоскостью в диаметральном направлении. Для нагружения на цилиндрической поверхности диска и нагружающей плоскости сгенерированы элементы контактного взаимодействия. При формировании модели использовались гексаэдрические элементы второго порядка SOLID186, а также элементы контактного интерфейса TARGET170 и CONTACT174 (терминология по [7]). В качестве контактного алгоритма использовался расширенный метод множителей Лагранжа.

Нагружение производилось кинематически, заданием равных предписанных перемещений узлам поверхности жёсткого штампа. При этом для восстановления кривой «нагрузка-перемещение», усилие снималось в виде суммарной силы реакции в узлах с предписанным перемещением по направлению, совпадающему с заданными перемещениями. С учётом симметрии, суммарное перемещение соответствует удвоенному текущему перемещению штампа (отражает результирующее сближение верхнего и нижнего штампа), а усилие - учетверённому суммарному усилию, снятому с узлов штампа (отражает факт того, что штамп в модели составляет $1 / 4$ часть полной площади штампа). Во всех проведённых расчётах конечное перемещение штампа составляло 50 мкм, что соответствует сближению штампов в 0,1 мм.

Решение в нелинейной постановке производилось для серии шагов - возрастающих значений предписанных перемещений с поиском равновесного состояния на каждом шаге алгоритмом Ньютона-Рафсона и обращением матрицы жёсткости методом сопряжённых градиентов с предобуславливанием.

\section{3. Результаты МКЭ моделирования}

На рис. 1 показаны картины распределения первого и третьего главных напряжений и максимальных касательных напряжений. Картины напряжений на рисунках получены при максимальном расчётном значении внедрения штампа - 50 мкм (сближение 0,1 мм), и абсолютные значения напряжений представлены только в целях иллюстрации их распределения.
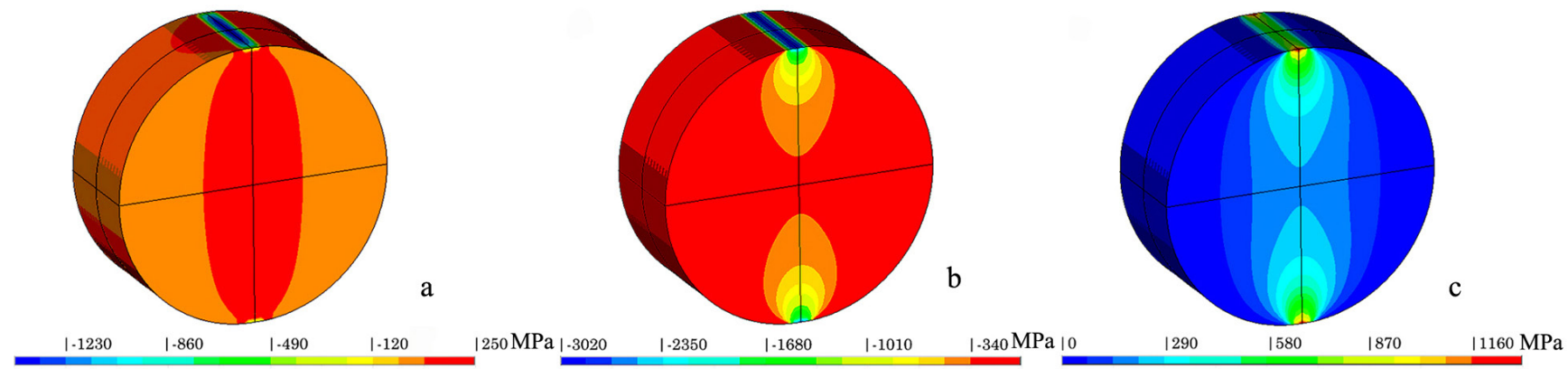

Pис. 1. Изоповерхности компонент напряжённого состояния: (a) $-\sigma_{1}$, (b) $-\sigma_{3}$, (c) $-\tau_{\max }$.

Fig. 1. Isosurfaces of the component of the stress state: (a) $-\sigma_{1}$, (b) $-\sigma_{3}$, (c) $-\tau_{\text {max }}$. 
Представленные распределения напряжений являются характерными для решения описанной задачи практически во всём диапазоне нагружения, за исключением малой начальной области нагружения. Это означает, что при возрастании предписанных перемещений жёсткого штампа от нуля до конечного значения, картина распределения напряжений по телу не меняется. Основным изменением характера распределения является смещение зоны максимальных растягивающих напряжений по направлению к центру диска. Не учитывались напряжения, действующие в области непосредственно примыкающей к контактной зоне, так как решение в этой области не является удовлетворительным с точки зрения точности величин и корректности описания по ряду критериев. На главных площадках во всём объёме образца действуют преимущественно сжимающие напряжения. Исключением является плоскость симметрии ZY. На этой и близлежащих параллельных плоскостях первые главные напряжения являются положительными и по направлению совпадают или близки с направлением $X\left(\sigma_{1} \approx \sigma_{x}\right)$. Феноменологически, при испытании дисковых образцов на диаметральное сжатие, разрушение происходит по диаметру, совпадающему с направлением сжатия. Сопоставление картины распределения напряжений с фактической картиной разрушения позволяет сделать ожидаемый вывод, что разрушение происходит под действием максимальных растягивающий напряжений, хотя зарождение разрушения возможно под действием максимальных касательных напряжений.

Максимальные растягивающие напряжения локализованы вблизи наружной поверхности диска и ориентированы в направлении, перпендикулярном направлению сжатия. При этом, положение точки максимума в процессе нагружения не постоянно и смещается в плоскости симметрии $Z Y$ от начального положения, в непосредственной близости от контактной зоны, по направлению к центру диска. Локализация максимальных растягивающих напряжений вблизи свободной поверхности, по всей видимости, связана с краевым эффектом. Крайний слой диска работает в условиях плоского напряжённого состояния, обусловленного краевыми условиями. Картина распределения напряжений показывает, что этот и ближайшие к нему слои являются более «жёсткими» по уровню действующих напряжений при нагружении по описанной здесь схеме. Вследствие этого, для определённых соотношений размеров диаметра и толщины диска, картина распределения напряжений может быть иной, что обуславливает необходимость ограничения допустимых соотношений диаметра и толщины при проведении испытаний. Стандартом ASTM D3967-95a предписывается определять разрушающие напряжения по формуле:

$$
\sigma_{t}=\frac{2 P}{\pi L D}
$$

где $P$ - нагрузка, при которой произошло разрушение, $L-$ толщина образца, $D$ - его диаметр. Сравним параметр напряжений, введённый стандартом, с первыми главными напряжениями, определёнными в нескольких различных точках, а именно: максимальные первые главные напряжения, действующие во всём объёме образца, первые главные напряжения, действующие в геометрическом центре объёма образца, и первые главные напряжения, действующие в геометрическом центре круглой поверхности образца. Согласно сделанным ранее выводам, эти напряжения ориентированы по диаметру диска, перпендикулярному вертикальной плоскости симметрии.

На рис. 2 представлены графики зависимости этих напряжений от параметра напряжений $\sigma_{t}$. Для чистоты рисунка результаты представлены только для образцов 4 различных размеров, характеристики упругости материала при этом принимались одинаковыми. По горизонтальной оси отложен параметр напряжений $\sigma_{t}$, подсчитанный, с учетом действующей на образец силы, по формуле (1), а по вертикальной - расчётное значение первых главных напряжений.

Анализируя рис. 2, можно сделать ряд заключений. Очевидно, что зависимость значения $\sigma_{1}^{\max }$ (максимального значения $\sigma_{1}$ ) от $\sigma_{t}$ не является линейной, общий вид зависимости близок ветви параболы относительно оси $\sigma_{t}$. При этом необходимо отметить, что вид кривой зависит от соотношения размеров диска, причём диски размером $10 \times 1,10 \times 2$ и $10 \times 3$ мм (приведена кривая для диска $10 \times 2$ ) имеют отличающиеся распределения, а зависимости, соответствующие остальным типоразмерам, незначительно отличаются друг от друга. Зависимости $\sigma_{1}$ от $\sigma_{t}$ в центре объёма и центре поверхности образца близки к линейным. Величина $\sigma_{1}$ в центре объёма образца превышает параметр $\sigma_{t}$, а величина $\sigma_{1}$ в центре поверхности образца ниже параметра $\sigma_{t}$ во всём диапазоне. Таким образом, можно сделать вывод, что, если разрушение материала происходит при достижении максимальными растягивающими напряжениями некоего предельного значения, то значение этого предельного напряжения не соответствует значению параметра $\sigma_{t}$, определённому по методу ASTM D3967-95a. Если же разрушение происходит по достижении средних напряжений в некотором сечении предельного значения, что характерно для неоднородных материалов - таких как бетон, или горные породы, параметр $\sigma_{t}$ является хорошей оценкой первого главного напряжения, усреднённого по оси $X$ диска.

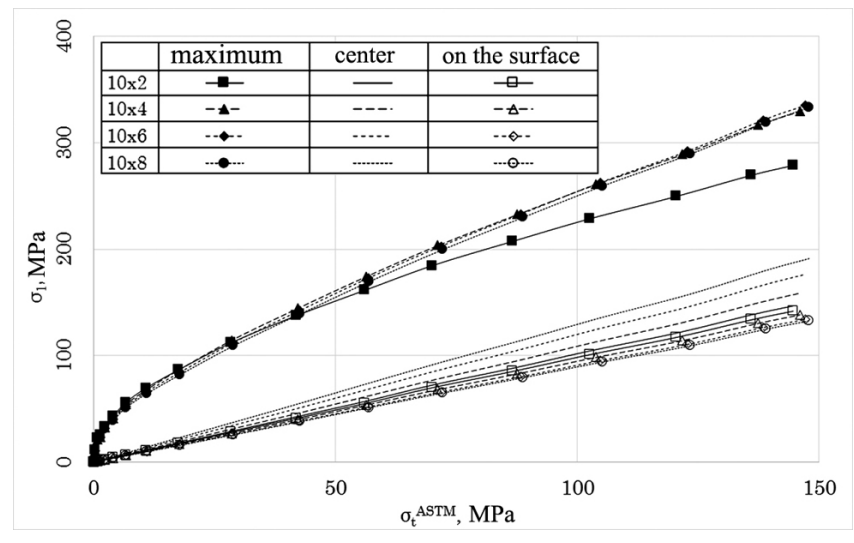

Рис. 2. Зависимость значения $\sigma_{1}$ в различных точках от $\sigma$ при варьировании толщины диска.

Fig. 2. The dependence of the values of $\sigma_{1}$ at different points from $\sigma_{t}$ by varying the thickness of the disc. 


\section{4. Результаты экспериментального исследования}

Экспериментальному исследованию подвергались дисковые образцы из серого чугуна и графита. Эти материалы отличаются прочностью и модулем упругости. Оба материала - хрупкие. В таблице 1 представлены механические свойства и модули упругости испытанных материалов.

\section{1. Испьтания дисков из серого чугуна}

Диски из серого чугуна были трех типоразмеров $D \times L$ : $10 \times 4,10 \times 5$ и $15 \times 4$ мм. Нагружение образцов (дисков) осуществлялось на испытательной машине INSTRON в специальном приспособлении, обеспечивающем строгую параллельность опорных плоскостей. Одновременно с нагружением образцов с помощью метода ЦКИ [9, 10] и применением установки Vic-3D RT осуществлялась регистрация перемещений точек поверхности каждого образца. Картины распределения деформаций єх поверхностных точек чугунного образца на начальной стадии нагружения и стадии, предшествующей разрушению, приведены на рис. 3a,b.

Распределение деформаций растяжения $\varepsilon_{x}$ (зоны красного цвета - максимальные деформации) точно соответствуют картине распределения растягивающих напряжений на рис. 1, что говорит об идентичности результатов, полученных расчетным и экспериментальным методами. Симметричные картины распределения деформаций относительно осей $X$ и $Y$ наблюдаются на всех стадиях нагружения образцов. В области контакта штампа и диска в поверхностных точках реализуется плоское напряженное состояние с главными растягивающими напряжениями $\sigma_{x}$ и сжимающими напряжениями $\sigma_{y}$, и, как следствие, значительными касательными напряжениями, равными полусумме абсолютных значений главных растягивающих и сжимающих напряжений. Касательные напряжения вызывают появление трещин сдвига, которые, соединяясь, приводят к разрушению образца в диаметральной плоскости, где действуют максимальные растягивающие напряжения, что подтвер- ждается характером разрушения образца. Ступенчатый излом в диаметральной плоскости образца свидетельствует о том, что и разрыв образца происходит под действием касательных напряжений. Не отмечено взрывного разрушения, характерного для хрупкого разрыва от нормальных напряжений. Наблюдали медленный отход одной половины образца от другой, что обычно присуще пластическому разрушению. Начало разрушения образцов связано с максимальными нагрузками, зафиксированными испытательной машиной. В таблицу 2 сведены результаты испытания 9-ти дисков из чугуна: максимальные нагрузки, соответствующие началу разрушения всех испытанных образцов, и разрушающие напряжения, подсчитанные по формулам (1) и (2)

$$
\sigma=\frac{P}{L D}
$$

Средние значения напряжений, подсчитанных по формуле (1), оказались близки значениям прочности материала на растяжение. Таким образом, для подсчета разрушающих напряжений при испытании металлических хрупких дисков по схеме «бразильского теста» может быть использована формула (1), рекомендуемая стандартом ASTM D3967-95a.

Табл. 1. Значения предела прочности при растяжении/сжатии и модуля Юнга для исследуемых материалов.

Table 1. The values of ultimate strength in tension/compression and Young's modulus of the materials studied.

\begin{tabular}{|c|c|c|c|}
\hline $\begin{array}{c}\text { Материал } \\
\text { Мaterial }\end{array}$ & $\begin{array}{c}\sigma_{\mathrm{s}}^{\mathrm{p}}, \mathrm{MПа} \\
\sigma_{\mathrm{s}}^{\mathrm{t}}, \mathrm{MPa}\end{array}$ & $\begin{array}{c}\sigma_{\mathrm{s}}^{\mathrm{c}}, \mathrm{MПа} \\
\sigma_{\mathrm{s}}^{\mathrm{c}}, \mathrm{MPa}\end{array}$ & $\begin{array}{c}E, \text { ГПа } \\
E, \mathrm{GPa}\end{array}$ \\
\hline $\begin{array}{c}\text { Чугун СЧ 10-40 } \\
\text { Cast iron SCH 10-40 }\end{array}$ & 104 & 404 & 100 \\
\hline $\begin{array}{c}\text { Графит APB-1 } \\
\text { Graphite ARV-1 }\end{array}$ & 13,8 & 37 & 11 \\
\hline
\end{tabular}
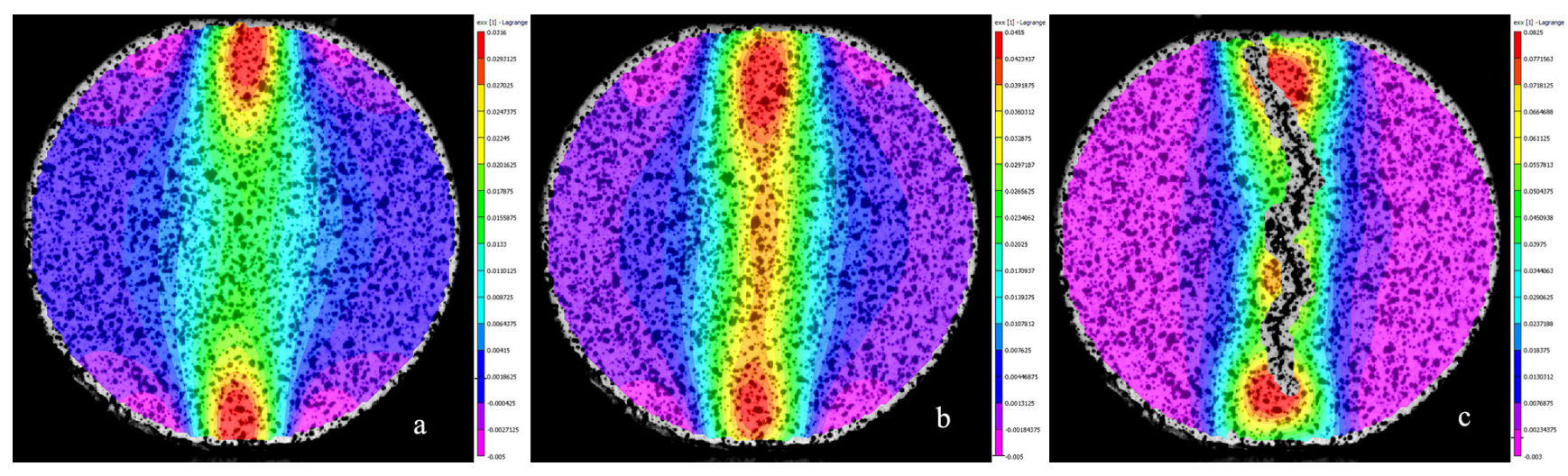

Рис. 3. Цветовые картины распределения деформаций $\varepsilon_{x}$ в поверхностных точках чугунного образца: (а) - начальная стадия нагружения, (b) - стадия, предшествующая разрушению, (c) - стадия разрушения.

Fig. 3. The color pattern of the strain distribution $\varepsilon_{x}$ in the surface of iron sample points: $(a)-$ the initial stage of loading, the (b) - stage preceding the destruction, (c) - stage destruction. 


\section{2. Испытания дисков из графита}

Диски из графита были двух типоразмеров $D \times L: 20 \times 10$ и $10 \times 5$ мм. Разрушение графитового диска происходит при максимальной нагрузке. Цветовые картины распределения деформаций $\varepsilon_{x}$ точно повторяют картины распределения деформаций в образце из чугуна и совпадают с результатами расчетного анализа. В графитовом образце размером $20 \times 10$ мм также выявлены начальные трещины в контактной области, которые служили источником разрушения образца. В таблице 3 приведены результаты испытания графитовых образцов и сопротивление графита разрушению, подсчитанное по формулам (1) и (2).

Полученные значения прочности графита на растяжение, подсчитанные по формуле (1), оказались на $20 \%$ ниже истинного (см. табл. 1), что можно считать вполне приемлемым оценочным результатом.

табл. 2. Сопротивление чугуна разрушению, определенное по формулам (1) и (2).

Table 2. The resistance of cast iron fracture, defined by the formulas (1) and (2).

\begin{tabular}{|c|c|c|c|c|}
\hline № образца & $\begin{array}{c}\text { Размер } \\
\text { образца, мм }\end{array}$ & $P, \mathrm{H}$ & $\sigma_{t}, \mathrm{M \Pi а}$ & $\sigma, \mathrm{MПа}$ \\
\hline No. sample & $\begin{array}{c}\text { The sample size, } \\
\mathrm{mm}\end{array}$ & $P, \mathrm{~N}$ & $\begin{array}{c}\sigma_{t}, \mathrm{MPa} \\
\text { (1) }\end{array}$ & $\begin{array}{c}\sigma, \mathrm{MPa} \\
(2)\end{array}$ \\
\hline 1 & $10 \times 4$ & 5333 & 85,3 & 134 \\
\hline 2 & $10 \times 4$ & 6028 & 96,2 & 151 \\
\hline 3 & $10 \times 4$ & 6979 & 111 & 174 \\
\hline 4 & $10 \times 4$ & 6847 & 109 & 171 \\
\hline 5 & $10 \times 4$ & 7029 & 112 & 176 \\
\hline 6 & $10 \times 5$ & 9009 & 115 & 180 \\
\hline 7 & $10 \times 5$ & 8783 & 112 & 176 \\
\hline 8 & $15 \times 4$ & 8290 & 87,9 & 138 \\
\hline 9 & $15 \times 4$ & 7550 & 80,2 & 126 \\
\hline \multicolumn{3}{|c|}{$\begin{array}{c}\text { Средние значения } \\
\text { Average values }\end{array}$} & 101 & 158,4 \\
\hline
\end{tabular}

табл. 3. Сопротивление графита разрушению, подсчитанное по формулам (1) и (2).

Table 3. The resistance of graphite fracture, calculated according to the formulas (1) and (2).

\begin{tabular}{|c|c|c|c|c|}
\hline Noo образца & $\begin{array}{c}\text { Размер } \\
\text { образца, мм } \\
\text { The sample size, } \\
\mathrm{mm}\end{array}$ & $P, \mathrm{~N}$ & $\begin{array}{c}\sigma_{t}, \mathrm{MPa} \\
(1)\end{array}$ & $\begin{array}{c}\sigma, \mathrm{MPa} \\
(2)\end{array}$ \\
\hline 1 & $20 \times 10$ & 3294 & 10,5 & 16,5 \\
\hline 2 & $20 \times 10$ & 3469 & 11,0 & 17,3 \\
\hline 3 & $10 \times 5$ & 855 & 10,9 & 17,1 \\
\hline 4 & $10 \times 5$ & 882 & 11,2 & 17,6 \\
\hline \multicolumn{2}{|c|}{$\begin{array}{c}\text { Средние значения } \\
\text { Average values }\end{array}$} & 10,9 & 17,1 \\
\hline
\end{tabular}

Благодарность/Acknowledgements. Статья написана в рамках программы по повышению конкурентоспособности НИЯУ «МИФИ».

\section{5. Заключение}

Таким образом, расчетным путем, с помощью МКЭ, и экспериментально с использованием метода ЦКИ, продемонстрировано полное соответствие распределений напряжений и деформаций в расчетной модели и результатов испытания дисковых образцов, нагружаемых по схеме “бразильского теста". Показана возможность косвенного определения прочности на растяжение материалов негеологического происхождения по результатам испытании малоразмерных дисковых образцов по схеме “бразильского теста” и использовании расчетной формулы, рекомендуемой стандартом ASTM D3967-95a.

\section{Литература/References}

1. E. G. Grigoryev. International journal «Machines, technologies, materials“. V.8, P.8 - 10. (2011).

2. S.S. Bashlykov, D.V. Demenyuk, E.G. Grigoryev, E.A. Olevsky, M.S. Yurlova. Physics and chemistry of materials processing. No. 5, p. 77 - 83. (2013). (in Russian) [C.С. Башлыков, В.Д. Деменюк, Е.Г. Григорьев, Е.А. Олевский, М. С. Юрлова. Физика и химия обработки материалов. № 5, С. 77 - 83. (2013).]

3. A. T. Tumanov. Methods of testing, control and research of engineering materials (Handbook). T. II. Methods of investigation of mechanical properties of metals. M.: Mashinostroenie, 1974.320 p. (in Russian) [А. Т. Туманов. Методы испытания, контроля и исследования машиностроительных материалов (Справочное пособие). T. II. Методы исследования механических свойств металлов. М.: Машиностроение, 1974. 320 с.]

4. A.G. Zholnin, I. V. Kovaleva, O.I. Pahilo - Darial, I. S. Litvinov, E. G. Grigoryev, E. A. Olevsky, V. Y. Goltsev, R. V., Rytenko. Physics and chemistry of materials processing. No. 2, p. 5-10. (2016). (in Russian) [А. Г. Жолнин, И. В. Ковалева, И. О. Пахило - Дарьял, И.С. Литвинова, Е.Г. Григорьев, Е.А. Олевский, В. Ю. Гольцев, В.Р. Рытенко. Физика и химия обработки материалов. № 2, С. 5 - 10. (2016).]

5. Q.Z. Wang, X.M. Jiaa, S.Q. Koub, Z.X. Zhangh, P. A. Lindgvistg. Int. J. of Rock Mechanics and Mining Sciences. V. 41, Issue 2, February. P.245-253. (2004).

6. ASTM D3967-95a. Standard Test Method for Splitting Tensile Strength of Intact Rock Core Specimens.

7. Release 16.2 Documentation for ANSYS [electronic document]/ANSYS Inc. Electronic data and software (104019 files: 10660130531 bytes).

8. NAFEMS search engineering analysis and simulation FEA, Finite Element Analysis, CFD, Computational Fluid Dynamics and Simulation. NAFEMS Ltd., Hamilton, United Kingdom.

9. M. A. Sutton, J. J. Orteu, H. W. Schreier. Image correlation for shape, motion and deformation measurements: basic concepts, theory and applications. Springer. P.321. (2009).

10. T.C. Chu, W.F. Ranson, M.A. Sutton, W.H. Peters. Experimental mechanics. V.25 (3). P.232 - 245. (1985). 